\title{
Viewpoints: Mathematical Perspective and Fractal Geometry in Art by Marc Frantz and Annalisa Crannel
}

\author{
Elena Marchetti
}

Published online: 21 February 2015

(C) Kim Williams Books, Turin 2015

\begin{abstract}
Elena Marchetti reviews book Viewpoints-Mathematical Perspective and Fractal Geometry in Art by Marc Frantz and Annalisa Crannel
\end{abstract}

Keywords Perspective $\cdot$ Fractals $\cdot$ Mathematical art $\cdot$ Mathematics education

This book is the result of the author's experience in teaching courses in mathematics and art. It is based on a collection of lessons coming from the long and active collaboration between the two authors, both mathematicians.

The text is intended for high school art students who must address the issue of perspective and its applications in different areas, and must also become familiar with fractals. It is divided into several chapters that cover topics related to the study of both perspective and fractal geometry. The sequence of chapters is such that their content is of increasing difficulty and all of them are structured in a similar way. Many chapters begin by dealing with a practical experiment that can be done with simple, everyday life tools. The authors invite teachers and students to organize such activities and reflect, each time, on their meaning. These experiments are in fact the necessary starting point that leads to the deduction and understanding of a theoretical synthesis. Each chapter is also coupled with several application exercises. The complete solution to some of them is given at the end of the book.

The book begins by addressing perspective. In the first two chapters, simple basic rules of analytic three-dimensional geometry are introduced; while the following chapters are devoted to one-point perspective and multiple-point perspective, highlighting the geometric properties of their construction. Projections of figures on

E. Marchetti $(\square)$

Dipartimento di Matematica "Francesco Brioschi",

Politecnico di Milano, Piazza Leonardo da Vinci, 32, 20133, Milan, Italy

e-mail: elena.marchetti@polimi.it 
spherical surfaces are then dealt with, in an elementary but effective way, in order to introduce the study of anamorphic images (Fig. 1).

The second part of the book is dedicated to fractal geometry. This topic is not simple. However, the authors manage to approach it in an intuitive yet rigorous way, highlighting the iterative process which characterizes the genesis of a fractal. The in-depth study of the subject is completed by addressing the problem of fractal dimension from a mathematical point of view: a topic which-I believe-is quite difficult to make clear to the kind of students for whom the book is written.

The level of the students for whom the text is intended led the authors to approach the mathematical examination of perspective avoiding in-depth investigation. In fact, simple and elementary notions (Cartesian coordinate system, proportions, properties of basic geometric forms, measures of distances and angles, geometric transformations) are sufficient to reach the level of mathematical knowledge required in most of the chapters. In contrast, the chapters devoted to fractals require knowledge of exponential and logarithmic functions.

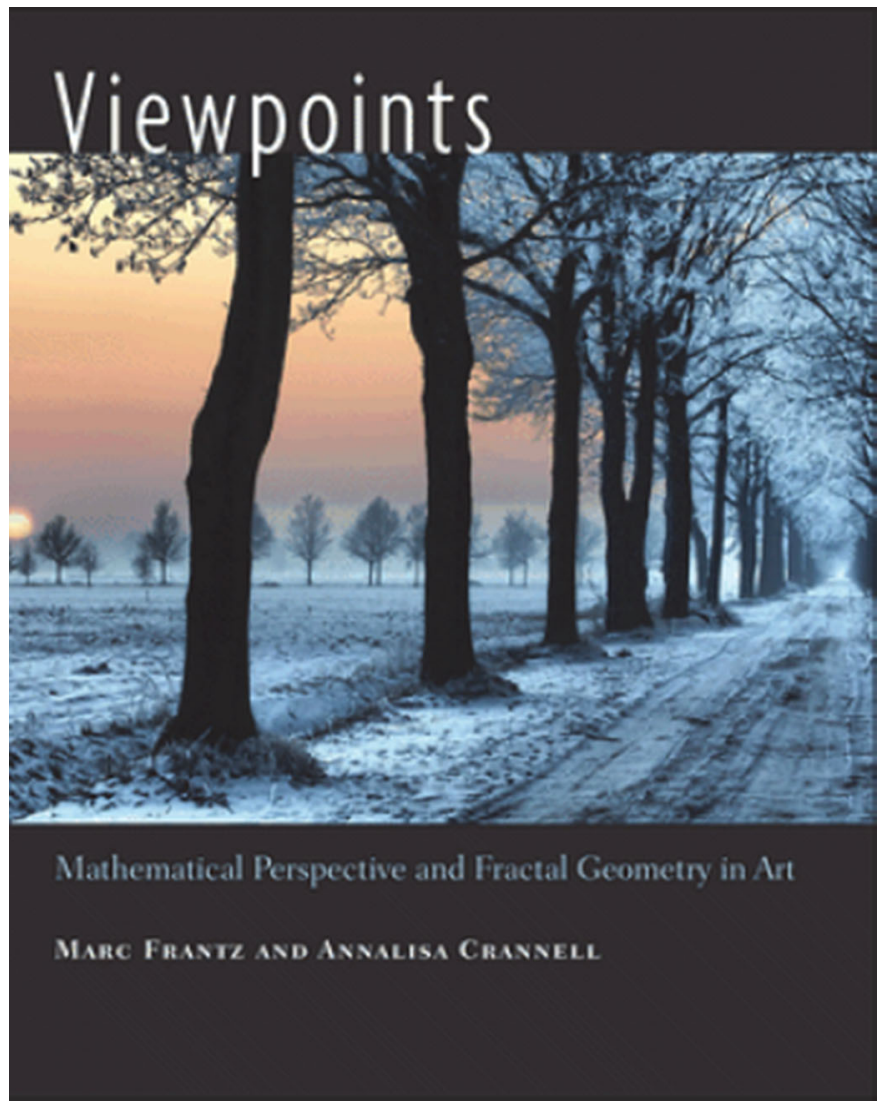

Fig. 1 Cover, Viewpoints: Mathematical Perspective and Fractals in Art 
The text is illustrated by many beautiful images both of artworks related to the subject matter, and pictures merely showing the different methods of construction of perspective views and fractals. The authors' personal interest in the use and application in the visual arts of the mathematical topics dealt with in the book is obvious.

The choice to insert in each chapter an "interview" with an artist or a scholar of perspective or fractals is original and much appreciated. Since the text is aimed at a very young audience, these interviews make the topic "modern" and are likely to motivate and encourage the young students to learn.

Viewpoints is indeed a textbook, but the publication layout, the colloquial tone with which the authors address the topics, and the organization of the chapters are such that the reader does not give the impression that one is reading an "old style" book. Clearly the book is designed to encourage students to learn how to handle perspective and fractals skillfully, and be able to draw by hand thanks to the understanding of their theoretical bases. In keeping with the purpose of promoting an active learning, no exercises based on the passive use of computer programs are included.

This is certainly an interesting book for teachers as well, since it shows an original work structure. The insertion in the appendix of some suggestions meant for teachers is a very good idea. Suggestions are related to the authors' personal experiences and their attention to teaching techniques, giving advice on how to organize a course by making the most of the contents of the book.

Viewpoints-Mathematical Perspective and Fractal Geometry in Art

by Marc Frantz and Annalisa Crannel

Princeton University Press, 2011

Elena Marchetti Associate Professor of "Istituzioni di Matematica", has taught since 1988 in Mathematics courses at the School of Architecture and Society of Politecnico di Milano (Italy). She has produced numerous publications in Italian and international scientific journals in the area of numerical integration and its applications. She has also published many papers about the links between mathematics, architecture and art. The experience gained through intense years of teaching courses to architecture students led her to collaborate in editing books dedicated to this topic, with multimedia support packages. 